\title{
Analytical solution for mixed convection and MHD flow of electrically conducting non-Newtonian nanofluid with different nanoparticles: A comparative study
}

\author{
Bhuvnesh Sharma $^{1 *}$, Sunil Kumar ${ }^{2}$, M.K. Paswan ${ }^{1}$ \\ ${ }^{1}$ Department of Mechanical Engg., National Institute of Technology, Jamshedpur 831014, Jharkhand, India \\ ${ }^{2}$ Department of Mathematics, National Institute of Technology, Jamshedpur 831014, Jharkhand, India
}

Corresponding Author Email: skiitbhu28@gmail.com

https://doi.org/10.18280/ijht.360327

Received: 23 February 2018

Accepted: 1 September 2018

\section{Keywords:}

homotopy analysis method (ham), magnetic parameter, MHD flow,

Nanofluid, ordinary differential equation

(ode) sodium alginate

\begin{abstract}
A rigorous analysis of unsteady electrically conducting nanofluid with MHD effect is presented. First, the governing partial differential equations for momentum and energy conservation are converted to couple nonlinear ordinary differential equations by means of exact similarity transformation. The Tiwari-Das nanofluid model is employed to obtain the analytical approximations for flow velocity and temperature distributions of alumina-sodium alginate nanofluid using HAM. The solution is found to be dependent on some parameters including the nanoparticle volume fraction, unsteadiness parameter, magnetic parameter, mixed convection parameter and the generalized Prandtl number. A systematic study is carried out to illustrate the effects of these parameters on the velocity and temperature distributions. Also, the value of skin friction coefficient and local Nusselt number are evaluated with variation of Prandtl number and compared with different nanoparticles.
\end{abstract}

\section{INTRODUCTION}

Nanofluid is formed by colloidal suspension of nanometersized solid particles (1-100nm diameter) into conventional liquids such as water, ethylene glycol, or oil. Firstly, the word "nanofluid"' was introduced by Choi [1-2], that represent the new class of fluid in which nanometer-scale particles are dispersed into conventional liquids. The thermal conductivity of base fluid can be increased up to 40 percent with low concentration ( $1-5 \%$ by volume) of nanoparticles in order to acheive higher heat transfer efficiency [3-4]. Xuan [3] increased the thermal conductivity of copper-water nanofluid upto $43 \%$ using hot wire method. Polyvinyl pyrrolidone, laurate salt and oleic acid are used as stabilizer to increase the stability of nanofluid [5-6].

Such flow occurs in various fluid engineering applications including submarine flow, turbo-machinery, aerofoil and oil ships. Hiemenz [7] introduced the stagnation point flow on the solid surface and reduced the Navier-Stokes equation into nonlinear ODE using similarity transformation. The study of stagnation flow in viscous boundary-layer in two-dimensional or axisymmetric stagnation region has gained attention by several researchers which have studied in the literature [8-11]. These studies were presented the basic behavior of viscous flow in stagnation region and applicability of similarity transformation with high accurate approximations.

Ramachandran et al. [12] presented the value of local Nusselt number and skin friction coefficient for laminar mixed convection flow of two-dimensional Navier-stokes equation with base fluid as water. Devi et al. [13] computed Nuxand Rex1/2Cfwith variation of $\operatorname{Pr}$ for mixed convective boundarylayer flow on vertical flat surface and Ishak et al. [14] showed same parameters with magnetohydrodynamic (MHD) effect on vertical surface flow with different Prandtl number. Later on, the same rheological problem studied for convective surface under the effect of magnetic field [15-16].

Sadoughi et al. [17] applied Reconstruction of Variational Iteration Method (RVIM) to find the solution of MHD boundary layer incompressible flow of $\mathrm{AL}_{2} \mathrm{O}_{3}$ nanofluid over a horizontal flat plate with base fluid as water and Alminiumoxide as nanoparticle. Amit and Habib-Olah [18] approximate analytical solutions of the MHD flow and heat transfer of a nanofluid using the differential transform method and Padè approximation method. Nandeppanavar [19] presented the analytical solutions for nonlinear boundary value problem under effects of the various governing parameters for the cases of $\mathrm{Cu}$-water nanofluid and the $\mathrm{Ag}$-water nanofluid.

HAM overcomes the limitations of perturbation methods as it provides freedom to choose an auxiliary parameter ( $\mathrm{h})$ which leads to increase in the convergence results. The solution to a condensation film in three dimensions on an inclined rotating disk was analytically done by Rashidi et al. [28]. Ziabakhsh et al. [29] applied HAM to compute the solution of hydromagnetic viscous flow.

This paper presents the velocity and flow analysis with influence of various parameters viz. nanoparticle volume fraction, unsteadiness parameter, magnetic parameter, mixed convection parameter and the generalized Prandtl number (Pr) for sodium alginate nanofluid with different nanoparticles. The values of skin-friction coefficient and local Nusselt number for nanofluid are tabulated with different Prandtl number and compared from different nanoparticles as copper $(\mathrm{Cu})$ and titanium dioxide $\mathrm{TiO}_{2}$ for sodium alginate nanofluid. The residual error illustrates the simplicity and accuracy of HAM. 


\section{PROBLEM STATEMENT AND MATHEMATICAL MODEL}

The $\mathrm{x}$ axis is measured along the normal of wedge and in positive direction from the wedge to the nanofluid whereas $y$ axis is considered along the wedge surface. The flow velocity is considered by $\mathrm{V}_{\infty}=\mathrm{by} /(1-\mathrm{at})$ and the stretching/shrinking velocity of wedge is assumed by $\operatorname{vw}(y, t)=c y /(1-a t)$, where c denotes the stretching/shrinking rate with $c<0$ or $c>0$ for shrinking or stretching wedge surface condition respectively, $\mathrm{b}$ is constant and $\mathrm{a}>0$ shows the stagnation flow strength. The surface temperature $(\mathrm{Tw})$ is defined as $\mathrm{Tw}(\mathrm{y}, \mathrm{t})=\mathrm{T} \infty+\mathrm{T} 0 \mathrm{y} /(1-$ at)2. Lok et al. [30] showed that the assisting flow occurs due to the heated upper half plate and the reason of opposing flow is cooled lower half plate. That's why the flow move upward near the heated wedge and tends to move down near the cooled wedge, see figure 1.

Tiwari and Das [31] presented the MHD nanofluid model with the assumption that the base fluid (sodium alginate) is in thermal equilibrium with nanoparticle, the governing equations for mass, momentum and energy in cartesian coordinates are

$\frac{\partial u}{\partial x}+\frac{\partial v}{\partial y}=0$

$\frac{\partial v}{\partial t}+u \frac{\partial u}{\partial x}+v \frac{\partial v}{\partial y}=\frac{\mu_{n f}}{\rho_{n f}} \frac{\partial^{2} v}{\partial x^{2}}-\frac{1}{\rho_{n f}} \frac{d p}{d y}-\frac{\sigma F^{2}}{\rho_{n f}} v+$

$\frac{\varphi \rho_{s} \beta_{s}+(1-\varphi) \rho_{f} \beta_{f}}{\rho_{n f}} a_{g}\left(T-T_{\infty}\right)$,

$\frac{\partial T}{\partial t}+v \frac{\partial T}{\partial y}+u \frac{\partial T}{\partial x}=\alpha_{n f} \frac{\partial^{2} T}{\partial x^{2}}$

The appropriate boundary conditions are

$t<0: \quad u=v=0, \quad T=T_{\infty} \quad$ for any $\quad x, y$,

$t \geq 0: v=v_{w}(y, t), u=U_{w}^{*}(t), T=T_{w}(y, t)$ at $x=0,(4)$

$x \rightarrow 0: v \rightarrow v_{\infty}(y, t), \quad T \rightarrow T_{\infty}$.

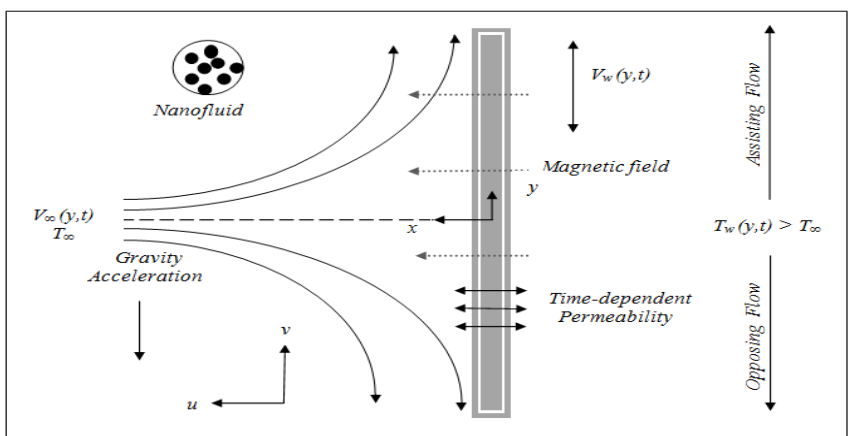

Figure 1. Coordinate system of flow configuration

Using generalized Bernoulli's equation, Eq. (2) will be as follows

$\frac{d v_{\infty}}{d t}+v_{\infty} \frac{d v_{\infty}}{d y}=-\frac{1}{\rho_{n f}} \frac{d p}{d y}-\frac{\sigma F^{2}}{\rho_{n f}} v_{\infty}$

$$
\begin{aligned}
& \frac{\partial v}{\partial t}+u \frac{\partial u}{\partial x}+v \frac{\partial v}{\partial y}=\frac{\mu_{n f}}{\rho_{n f}} \frac{\partial^{2} v}{\partial x^{2}}+\frac{d v_{\infty}}{d t}+v_{\infty} \frac{d v_{\infty}}{d t} \\
& +\frac{\sigma F^{2}}{\rho_{n f}}\left(v_{\infty}-v\right)+\frac{\phi \rho_{s} \beta_{s}+(1-\phi) \rho_{f} \beta_{f}}{\rho_{n f}} a_{g}\left(T-T_{\infty}\right)
\end{aligned}
$$

Table 1. Thermophysical properties of base fluid and nanoparticles [24]

\begin{tabular}{cccccc}
\hline & $\boldsymbol{C} \boldsymbol{P}$ & $\boldsymbol{\rho}$ & $\boldsymbol{k}$ & $\boldsymbol{\alpha \times 1 0 ^ { 7 }}$ & $\boldsymbol{\beta} \times \mathbf{1 0}^{-5}$ \\
\hline $\begin{array}{c}\text { Sodium } \\
\text { Alginate }\end{array}$ & 4175 & 989 & 0.6376 & 1.62 & 23 \\
\hline Copper & 385 & 8933 & 400 & 1163.1 & 1.67 \\
\hline $\begin{array}{c}\text { Titanium } \\
\text { dioxide }\end{array}$ & 686.2 & 4250 & 8.954 & 30.7 & 0.9 \\
\hline Alumina & 765 & 3970 & 40 & 131.7 & 0.85 \\
\hline
\end{tabular}

The viscosity ( $\mu n f)$, thermal diffusivity ( $\alpha n f)$, density ( $\rho n f)$ and heat capacitance ( $\rho \mathrm{Cp}) \mathrm{nf}$ of nanofluid are defined as

$$
\begin{aligned}
& \mu_{n f}=\frac{\mu_{f}}{(1-\phi)^{2.5}} \\
& \rho_{n f}=(1-\phi) \rho_{f}+\phi \rho_{s} \\
& \left(\rho C_{p}\right)_{n f}=(1-\phi)\left(\rho C_{p}\right)_{f}+\phi\left(\rho C_{p}\right)_{s} \\
& \alpha_{n f}=\frac{k_{n f}}{\left(\rho C_{p}\right)_{n f}}
\end{aligned}
$$

The thermal conductivity of nanofluid (knf) are given by Maxwell-Garnett model [32], which is presented as follows

$\frac{k_{n f}}{k_{f}}=\frac{\left(k_{s}+2 k_{f}\right)-2 \phi\left(k_{f}-k_{s}\right)}{\left(k_{s}+2 k_{f}\right)+\phi\left(k_{f}-k_{s}\right)}$

The development process of this model exhibit the transformation of governing equations to $n, \in$ system. The similar variable $n$ involves both $\mathrm{x}$ and $\mathrm{y}$, while $\in$ is related to $\mathrm{x}$ alone. Therefore, we assume $\in=0$ for any stream-wise location and $\mathrm{f}$ is the function of only variable $n$. To proceed, we adopt the following similarity transformations.

The physical stream function is introduced as

$\psi=\left(\frac{b u_{f}}{1-a t}\right)^{1 / 2} y f(\eta)$

The dimensionless temperature is defined as

$\theta(\eta)=\frac{T-T_{\infty}}{T_{w}-T_{\infty}}$

The transformed similar variable is

By substituting Eq. (5), Eq. (2) can be presented as 
$\eta=\left(\frac{b}{u_{f}(1-a t)}\right)^{1 / 2} x$

The stream function can be defined by

$u=\frac{\partial \psi}{\partial x}, v=-\frac{\partial \psi}{\partial y}$

Using stream function, the velocity component $u$ and $v$ can be derived as follows

$$
\begin{aligned}
& u=-\left(\frac{u b}{1-a t}\right)^{1 / 2} f(\eta) \\
& v=\frac{b y}{1-a t} f^{\prime}(\eta)=V_{\infty}(y, t) f^{\prime}(\eta)
\end{aligned}
$$

The surface mass flux $\mathrm{U}_{\mathrm{w}}{ }^{*}$ is transformed in term of wall transpiration parameter:

$$
U_{w}^{*}=-\left(\frac{u b}{1-c t}\right)^{1 / 2} U_{w}
$$

The mass conservation equation (1) is identically satisfied with stream function. Under the transformation (7)-(18), the momentum equation (6) and energy conservation equation (3) reduce to the following nonlinear ordinary differential equation:

$$
\begin{aligned}
& \frac{1}{(1-\phi)^{2.5}\left(1-\phi+\phi \frac{\rho_{s}}{\rho_{f}}\right)} f^{\prime \prime \prime}+f f^{\prime \prime}-f^{\prime 2}+1+ \\
& A\left(1-f^{\prime}-\frac{1}{2} \eta f^{\prime \prime}\right)+\frac{M}{\left(1-\phi+\phi \frac{\rho_{s}}{\rho_{f}}\right)}\left(1-f^{\prime}\right)+
\end{aligned}
$$

$\frac{1-\phi+\phi\left(\frac{\rho_{s}}{\rho_{f}}\right)\left(\frac{\beta_{s}}{\beta_{f}}\right)}{\rho_{s}} \lambda \theta=0$

$$
1-\phi+\phi \frac{\rho_{s}}{\rho_{f}}
$$

$$
\frac{\frac{k_{n f}}{k_{f}}}{(1-\phi)+\phi \frac{\left(\rho C_{p}\right)_{s}}{\left(\rho C_{p}\right)_{f}}} \theta^{\prime \prime}+
$$

$\operatorname{Pr}\left(f \theta^{\prime}-\theta f^{\prime}-A\left(2 \theta+\frac{1}{2} \eta \theta^{\prime}\right)\right)=0$

which are subjected to the transformed boundary conditions

$\eta \rightarrow 0: f=U_{w}, f^{\prime}=c / b=\in, \quad \eta \rightarrow \infty: f^{\prime}=1$ $\theta \rightarrow 0: \theta=1 \eta \rightarrow \infty: \theta=0$

The buoyancy or mixed convection parameter $\lambda$, local Grashof number $\left(\mathrm{Gr}_{\mathrm{y}}\right)$ and Reynold number are defined as

$\lambda=\frac{G r_{y}}{\operatorname{Re}_{y}}=a_{g} \beta_{f} c / b^{2}$

$G r_{y}=a_{g} \beta_{f}\left(T_{w}-T_{\infty}\right) \frac{y^{3}}{v_{f}^{2}}$

$\operatorname{Re}_{y}=\frac{V_{\infty} y}{v_{f}}$

The skin-friction coefficient is defined as

$C_{f}=\frac{\tau_{w}}{\rho f v^{2} / 2}$

and the local Nusselt number is defined as

$N u_{y}=\frac{y Q_{w}}{k_{f}\left(T_{w}-T_{\infty}\right)}$

where the wall shear stress $\tau_{w}$ can be written as

$\tau_{w}=\mu_{n f}\left(\frac{\partial v}{\partial x}\right)_{x=0}$

and the heat flux $Q_{w}$ is

$Q_{w}=-k_{n f}\left(\frac{\partial T}{\partial x}\right)_{x=0}$

Using similarity variables Eq. (12)-(17), the skin-friction coefficient and Nusselt number can be presented in the form

$C_{f}\left[\operatorname{Re}_{y}\right]^{1 / 2}=\frac{1}{(1-\phi)}$

$N u_{y}\left[\operatorname{Re}_{y}\right]^{-1 / 2}=-\frac{k_{n f}}{k_{f}} \theta^{\prime}(0)$

\section{HAM SOLUTIONS}

The initial guess $\mathrm{f}_{0}(n)$ and $\theta(n)$ of the transformed Eqs. (19) and (20) are choosen for HAM solutions as follows

$$
f_{0}(\eta)=U_{w}-(1-\in)+\eta+(1-\in) e^{-\eta}
$$


$\theta_{0}(\eta)=e^{-\eta}$

and we consider the linear operators:

$L_{f}=\frac{\partial^{3} f}{\partial \eta^{3}}-\frac{\partial f}{\partial \eta}$

$L_{\theta}=\frac{\partial^{2} \theta}{\partial \eta^{2}}-\theta$

Introducing a embedding parameter $\mathrm{q}$ and convergencecontrol parameter $h$, the zeroth-order deformation equations

$(1-q) L_{f}\left[f(\eta ; q)-f_{0}(\eta)\right]=q \hbar H_{f} N_{f}[f(\eta ; q)]$

$f(0 ; q)=U_{w}, f^{\prime}(0 ; q)=\in, \quad f^{\prime}(\infty ; q)=1$

$(1-q) L_{\theta}\left[\theta(\eta ; q)-\theta_{0}(\eta)\right]=q \hbar H_{\theta}(\eta) N_{\theta}[\theta(\eta ; q)]$

$\theta(0 ; q)=1, \theta^{\prime}(\infty ; q)=0$

For $q=0$ and $q=1$, we have respectively

$q=0: f(\eta ; 0)=f_{0}(\eta), \theta(\eta ; 0)=\theta_{0}(\eta)$

$q=1: f(\eta ; 1)=f(\eta), \theta(\eta ; 1)=\theta(\eta)$

$f(n ; p)$ varies from $\mathrm{f}_{0}(n)$ to $\mathrm{f}(n)$ and $\theta(n ; p)$ varies from $\theta_{0}$ $(n)$ to $\theta(n)$, when $q$ increases from 0 to 1 . Using Eqs. (51) (52) and Taylor's theorem, $f(n ; q)$ and $f(n ; q)$ can be presented in a power series form. In which the nonlinear operators are presented as

$$
\begin{aligned}
& N_{f}=\frac{1}{(1-\phi)^{2.5}\left(1-\phi+\phi \frac{\rho_{s}}{\rho_{f}}\right)}\left(\frac{\partial^{3} f(\eta ; q)}{\partial \eta^{3}}\right)+ \\
& f(\eta ; q)\left(\frac{\partial^{2} f(\eta ; \theta)}{\partial \eta^{3}}\right)+f(\eta ; q)\left(\frac{\partial^{2} f(\eta ; q)}{\partial \eta^{2}}\right)- \\
& +\left(1-\chi_{m}\right)+A\left(1-\frac{\partial f(\eta ; q)}{\partial \eta}-\frac{1}{2} \eta \frac{\partial^{2} f(\eta ; q)}{\partial \eta^{2}}-\chi_{m}\right)+ \\
& \frac{M}{\left(1-\phi+\phi \frac{\rho_{s}}{\rho_{f}}\right)}\left(\frac{\partial f(\eta ; q)}{\partial \eta}\right)+\frac{1-\phi+\phi\left(\frac{\rho_{s}}{\rho_{f}}\right)\left(\frac{\beta_{s}}{\beta_{f}}\right)}{1-\phi+\phi \frac{\rho_{s}}{\rho_{f}}} \lambda \theta
\end{aligned}
$$

$N_{\theta}=\frac{\frac{k_{n f}}{k_{f}}}{(1-\varphi)+\varphi \frac{\left(\rho C_{p}\right)_{s}}{\left(\rho C_{p}\right)_{f}}}\left(\frac{\partial^{2} \theta(\eta ; q)}{\partial \eta^{2}}\right)+$

$\operatorname{Pr}\left(f(\eta ; q) \frac{\partial \theta(\eta ; q)}{\partial \eta}-\theta(\eta ; q) \frac{\partial f(\eta ; q)}{\partial \eta}-\right.$

$A\left(2 \theta(\eta ; q)+\frac{1}{2} \eta \frac{\partial \theta(\eta ; q)}{\partial \eta}\right)$

$f(\eta ; q)=f_{0}(\eta)+\sum_{m=1}^{\infty} f_{m}(\eta) q^{m}$

$f_{m}(\eta)=\left.\frac{1}{m !} \frac{\partial^{m} f(\eta ; q)}{\partial q^{m}}\right|_{q=0}$

$\theta(\eta ; q)=\theta_{0}(\eta)+\sum_{m=1}^{\infty} \theta_{m}(\eta) q^{m}$

$\theta(\eta)=\left.\frac{1}{m !} \frac{\partial^{m} \theta(\eta ; q)}{\partial q^{m}}\right|_{q=0}$

In which the auxiliary parameter is selected such the series is convergent at $q=1$. Liao [26] pointed out that the convergenceregion depends on a convergence-control parameter $\hbar$.Then,

$f(\eta)=f_{0}(\eta)+\sum_{m=1}^{\infty} f_{m}(\eta)$

$\theta(\eta)=\theta_{0}(\eta)+\sum_{m=1}^{\infty} \theta_{m}(\eta)$

The \$m\$th-order deformation equations:

$L\left[f_{m}(\eta)-\chi_{m} f_{m-1}(\eta)\right]=\hbar H_{f}(\eta) R_{m}^{f}(\eta)$

$L\left[\theta_{m}(\eta)-\chi_{m} \theta_{m-1}(\eta)\right]=\hbar H_{\theta}(\eta) R_{m}^{\theta}(\eta)$

$\eta=0: f_{m}=U_{w}, f_{m}^{\prime}=\in, \eta=\infty: f_{m}=1$

$\theta=0: \theta_{m}=1, \theta=\infty: \theta_{m}=0$

where $R_{m}^{f}(n)$ and $R_{m}^{\theta}(n)$ are defined as 


$$
\begin{aligned}
& R_{m}^{f}(\eta)=\frac{1}{(1-\varphi)^{2.5}\left(1-\varphi+\varphi \frac{\rho_{s}}{\rho_{f}}\right)}\left(\frac{\partial^{3} f_{m-1}(\eta ; q)}{\partial \eta^{3}}\right) \\
& \quad\left[f_{n}(\eta ; q) \frac{\partial^{2} f_{m-1-n}(\eta ; q)}{\partial \eta^{2}}-\frac{\partial f_{n}(\eta ; q)}{\partial \eta} \frac{\partial f_{m-1-n}(\eta ; q)}{\partial \eta}\right] \\
& +\left(1-\chi_{m}\right)+ \\
& \quad A\left(1-\frac{\partial f_{m-1}(\eta ; q)}{\partial \eta}-\frac{1}{2} \eta \frac{\partial^{2} f_{m-1}(\eta ; q)}{\partial \eta^{2}}-\chi_{m}\right) \\
& +\sum_{n=0}^{m-1}+\frac{M}{\left(1-\varphi+\varphi \frac{\rho_{s}}{\rho_{f}}\right)}\left(1-\frac{\partial f_{m-1}(\eta ; q)}{\partial \eta}\right) \\
& +\frac{1-\varphi+\varphi\left(\frac{\rho_{s}}{\rho_{f}}\right)\left(\frac{\beta_{s}}{\beta_{f}}\right)}{1-\varphi+\varphi \frac{\rho_{s}}{\rho_{f}}} \lambda \theta_{m-1}(\eta ; q)
\end{aligned}
$$

$$
\begin{aligned}
& R_{m}^{\theta}(\eta)=\frac{\frac{k_{n f}}{k_{f}}}{(1-\phi)+\phi \frac{\left(\rho C_{p}\right)_{s}}{\left(\rho C_{p}\right)_{f}}}\left(\frac{\partial^{2} \theta_{m-1}(\eta ; q)}{\partial \eta^{2}}\right)+\operatorname{Pr} \\
& \left(\sum_{n=0}^{m-1}\left[f_{n}(\eta ; q) \frac{\partial \theta_{m-1-n}(\eta ; q)}{\partial \eta}-\frac{\partial f_{n}(\eta ; q)}{\partial \eta} \theta_{m-1-n}(\eta ; q)\right]\right) \\
& -A\left(2 \theta_{m-1}(\eta ; q)+\frac{1}{2} \eta \frac{\partial \theta_{m-1}(\eta ; q)}{\partial \eta}\right)
\end{aligned}
$$

we consider the auxiliary function as $H_{f}(\eta), H_{\theta}(\eta)=1$. The calculation is made for sodium alginate nanofluid with alumina as nanoparticle with $P r=6.2$. The parameters of nanofluid are assumed as $A=0.5, \lambda=1, M=1, U_{w}=0.5$ and $\varphi=0.2$ to obtain the solution of coupled equations with appropriate boundary conditions.

$$
\begin{aligned}
& f_{1}(\eta)=-0.5+e^{-\eta}-1.5892 \hbar+1.5892 e^{-\eta} \hbar \\
& +1.5892 e^{-\eta} \hbar \eta+0.1875 e^{-\eta} \hbar \eta^{2}
\end{aligned}
$$

$$
\theta_{1}(\eta)=e^{-\eta}+4.9092 e^{-\eta} \hbar \eta+1.1625 e^{-\eta} \hbar \eta^{2}
$$

$$
f_{2}(\eta)=-1.5892 \hbar+1.5892 e^{-\eta} \hbar-7.5388 \hbar^{2}
$$$$
-0.0625 e^{-2 \eta} \hbar^{2}+7.6013 e^{-\eta} \hbar^{2}+1.5892 e^{-\eta} \hbar \eta+
$$$$
7.4763 e^{-\eta} \hbar^{2} \eta+0.1875 e^{-\eta} \hbar \eta^{2}+2.6405 e^{-\eta} \hbar^{2} \eta^{2}
$$$$
+0.35925 e^{-\eta} \hbar^{2} \eta^{3}+0.0175 e^{-\eta} \hbar^{2} \eta^{4}
$$

$$
\begin{aligned}
& \theta_{2}(\eta)=12.234 e^{-2 \eta} \hbar^{2}-12.234 e^{-\eta} \hbar^{2}+4.9092 e^{-\eta} \hbar \eta \\
& +4.03 e^{-2 \eta} \hbar^{2} \eta+24.0522 e^{-\eta} \hbar^{2} \eta+1.1625 e^{-\eta} \hbar \eta^{2} \\
& +14.6007 e^{-\eta} \hbar^{2} \eta^{2}+4.806 e^{-\eta} \hbar^{2} \eta^{3}+ \\
& 0.6757 e^{-\eta} \hbar^{2} \eta^{4}
\end{aligned}
$$

Similarly, the computation of 15 th order approximation of
HAM is executed on MATHEMATICA 7.0 software with BVPh 2.0 package. Convergence the of HAM solutions

\section{CONVERGENCE OF HAM SOLUTIONS}

The family of solutions presented by HAM is expressed in the form of an auxiliary parameter. The convergence region and rate of approximation strongly depends on the convergence-control parameter $h$, as stated by Liao [27]. Fig. 2 depicts the $h$-curves of dimensionless velocity \& temperature obtained from Eqs. (19) and (20) based on the 10th order approximation.

To find out an optimal value of a convergence-control parameter $\hbar$ the averaged residual square error can be written

$$
\begin{aligned}
& E_{l, f}=\frac{1}{K} \sum_{j=1}^{K}\left(N_{f}\left[\sum_{i=0}^{l} f_{i}(j \Delta x)\right]\right)^{2} \\
& E_{l, \theta}=\frac{1}{K} \sum_{j=1}^{K}\left(N_{\theta}\left[\sum_{i=0}^{l} \theta_{i}(j \Delta x)\right]\right)^{2}
\end{aligned}
$$

where $\Delta \mathrm{x}=10 / K, K=20$ for transformed Eqs. (19) and (20). The optimal value of $h$ is evaluated by minimizing the average residual square error $E_{m}$ corresponding to the transformed nonlinear equations

$$
\frac{d E_{l, f}}{d \hbar}=0
$$

$$
\frac{d E_{l, \theta}}{d \hbar}=0
$$

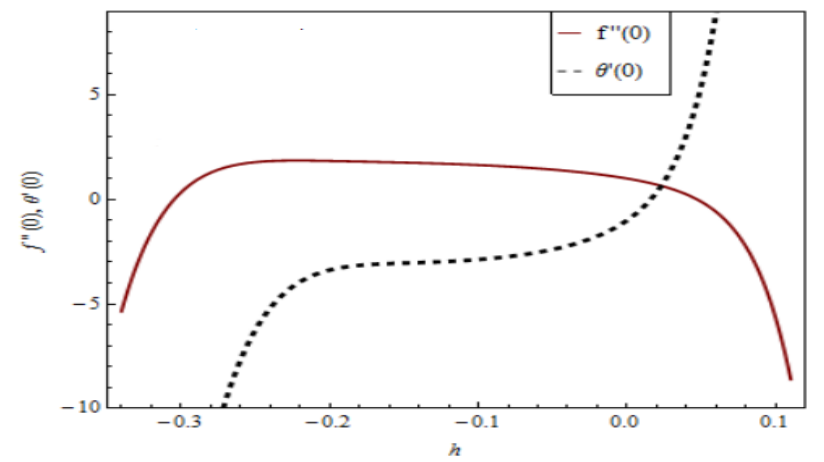

Figure 2. The $h$ curves of dimensionless velocity and dimensionless temperature for alumina-sodium alginate nanofluid with $A=0.5, M=1, U_{w}=0.5, \varepsilon=0, P r=6.2, \varphi=$ $0.2, \lambda=1$

Table 2. Acceptable values of $h$ for alumina-sodium alginate nanofluid with $A=0.5, M=1, U_{w}=0.5, \operatorname{Pr}=6.2, \varphi=0.2, \lambda$ $=1$

\begin{tabular}{cc}
\hline Series & Acceptable range \\
\hline$f^{\prime}(\eta)$ & $-0.25 \leq \hbar \leq-0.05$ \\
$\theta(\eta)$ & $-0.18 \leq \hbar \leq-0.1$ \\
\hline
\end{tabular}


Table 3 exhibit the comparison of the averaged residual square error and optimal value of $h$ for velocity and temperature distributions of alumina-sodium alginate nanofluid with increasing the order of approximations.

Table 3. Optimal value of $\mathrm{n}$ for alumina-sodium alginate nanofluid with $A=0.5, M=1, U_{w}=0.5, P r=6.2, \varphi=6.2, \lambda$ $=1$

\begin{tabular}{ccccc}
\hline $\mathbf{N}$ & $\begin{array}{c}\boldsymbol{f}^{\prime}(\boldsymbol{\eta}) \\
\text { optimal }\end{array}$ & $\begin{array}{c}\boldsymbol{f}^{\prime}(\boldsymbol{\eta}) \\
\boldsymbol{E}_{l, f}\end{array}$ & $\boldsymbol{\theta}_{\text {optimal }} \hbar$ & $\boldsymbol{\theta}_{\boldsymbol{E}_{\boldsymbol{l}}, \boldsymbol{\theta}}$ \\
\hline 1 & -0.2432 & 0.68499 & -0.1729 & $2.1767 \times 10^{-2}$ \\
2 & -0.2385 & 0.16878 & -1.31615 & $0.1735 \times 10^{-2}$ \\
3 & -0.2174 & 0.15924 & -1.38356 & $0.1587 \times 10^{-2}$ \\
\hline
\end{tabular}

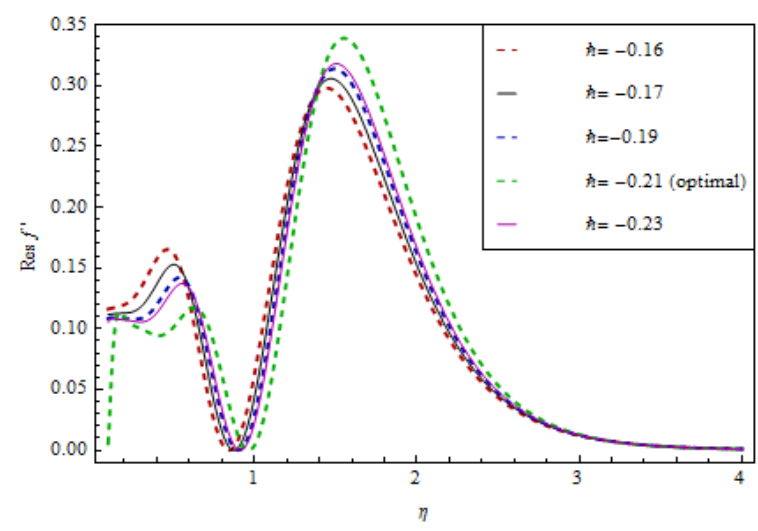

Figure 3. Residual error for non-dimensional velocity for alumina-sodium alginate nanofluid with $A=0.5, M=1$, $U_{w}=0.5, \varepsilon=0, \operatorname{Pr}=6.2, \varphi=0.2, \lambda=1$

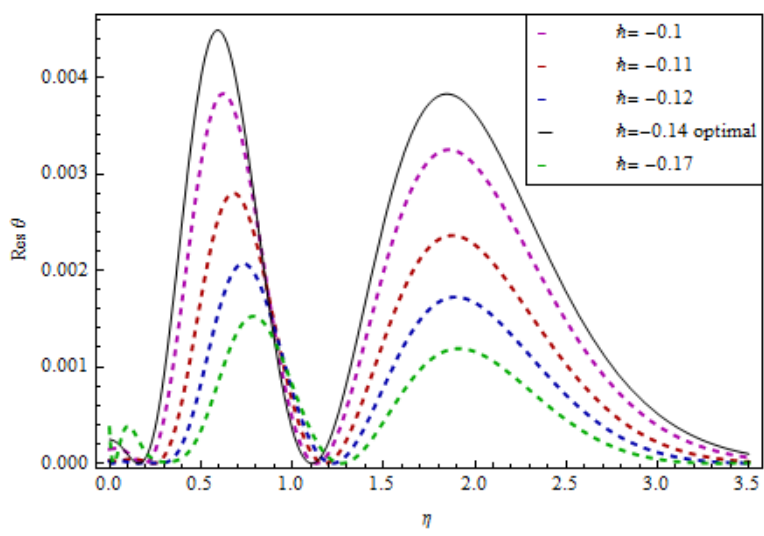

Figure 4. Residual error for non-dimensional temperature for alumina-sodium alginate nanofluid with $A=0.5, M=1, U_{w}=$ $0.5, \varepsilon=0, \operatorname{Pr}=6.2, \varphi=0.2, \lambda=1$

Table 4. The values of skin friction coefficient and local Nusselt number for alumina-sodium alginate nanofluid with various values of $P r$ when $A=0, \varepsilon=0, M=0, U_{w}=0, \varphi=0$,

$$
\lambda=1
$$

\begin{tabular}{|c|c|c|}
\hline $\boldsymbol{P r}$ & $\boldsymbol{R} e_{x}{ }^{1 / 2} C_{f}$ & $N u_{x} R e_{x-1 / 2}$ \\
\hline 0.7 & 1.8573 & 0.8521 \\
\hline 1 & 1.7520 & 0.8872 \\
\hline 7 & 1.7385 & 1.8036 \\
\hline 10 & 1.6214 & 2.0145 \\
\hline 20 & 1.5501 & 2.7326 \\
\hline 40 & 1.4623 & 3.2214 \\
\hline 50 & 1.3986 & 3.7048 \\
\hline
\end{tabular}

The accuracy and validity of the HAM can be demonstrated by the residual square error curves which are plotted in figure 3 and figure 4 for $f^{\prime}$ and $\theta$ with several values of auxiliary parameter for alumina-sodium alginate nanofluid. Table 4 exhibit the value of skin fiction coefficient and local Nusselt number for different values of Prandtl number in order to illustrate the validity and efficiency of HAM.

\section{RESULTS AND DISCUSSIONS}

HAM has been effectively applied to evaluate the analytical solution for transformed nonlinear ordinary differential equations (19)-(20) describing boundary-layer flow and heat transfer for MHD mixed convection nanofluids with some values including wall transpiration parameter $\left(\mathrm{U}_{\mathrm{w}}\right)$, mixed convection parameter $\lambda$, velocity ratio parameter $(\varepsilon)$, nanoparticle volume fraction $(\varphi)$, unsteadiness parameter $(A)$ and magnetic parameter $(M)$. In order to illustrates the effects of these parameters, the velocity and temperature profile has been presented from figures (5)-(16) for alumina-sodium alginate nanofluid using 15 th-order of approximation. The value of Prandtl numberis considered as 6.2 (for water) and the range of nanoparticle volume fraction parameter varies from 0 (Newtonian fluid) to 0.2 as pointed out by Oztop and AbuNada [32].

Table (5)-(7) present the values of skin friction coefficient and local Nusselt number with nanoparticle volume fraction $(\varphi)$ in case of stretching/shrinking sheet and asssisting/opposing flows. Tables reveal that value of skin friction coefficient and local Nusselt number are higher for $\mathrm{Cu}$ as nanoparticle compared to $\mathrm{Al}_{2} \mathrm{O}_{3}$ and $\mathrm{TiO}_{2}$ nanoparticles.

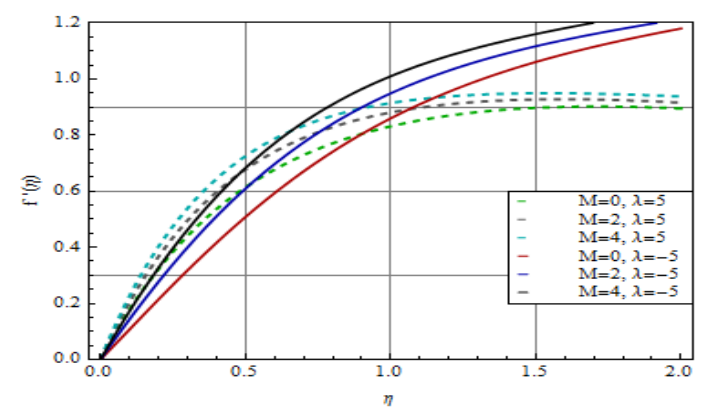

Figure 5. The velocity distribution for alumina-sodium alginate nanofluid for different values of mixed convection and magnetic parameters with $A=0.5$, $U_{w}=0.5, \varepsilon=0, \operatorname{Pr}=6.2, \varphi=0.2$

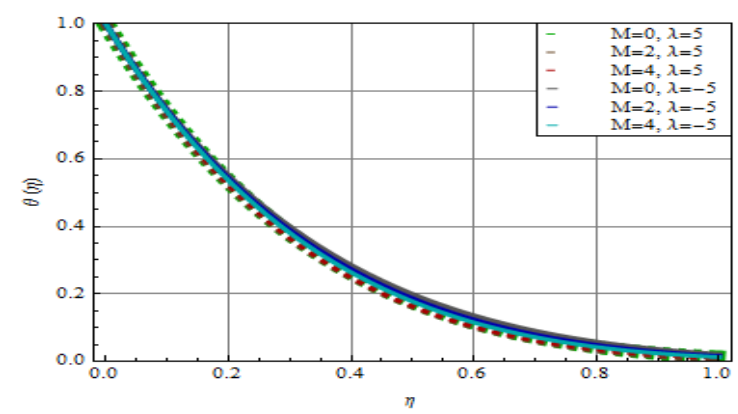

Figure 6. The temperature distribution for alumina-sodium alginate nanofluid for different values of mixed convection and magnetic parameters with $A=0.5$, $U_{w}=0.5, \varepsilon=0, \operatorname{Pr}=6.2, \varphi=0.2$ 


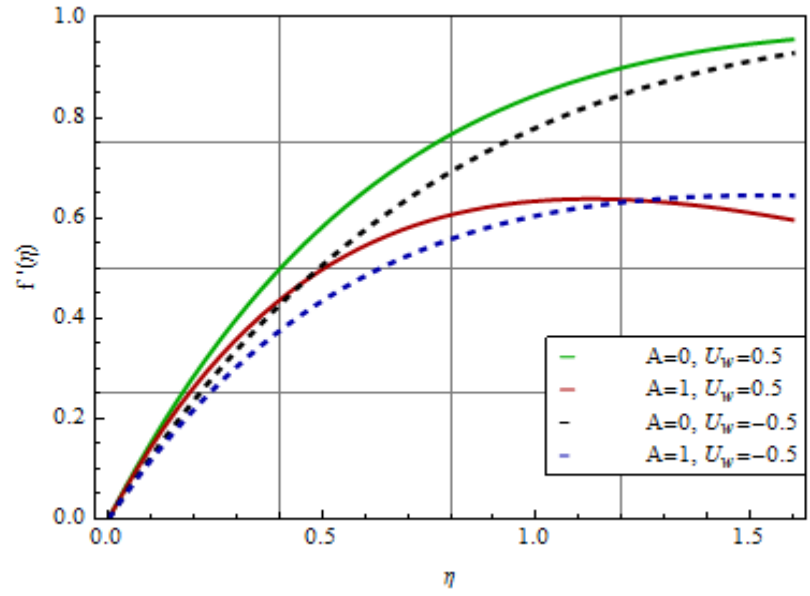

Figure 7. The velocity distribution for alumina-sodium alginate nanofluid for different values of mixed convection and unstediness parameters with $M=1$, $U_{w}=0.5, \varepsilon=0, \operatorname{Pr}=6.2, \varphi=0.2$

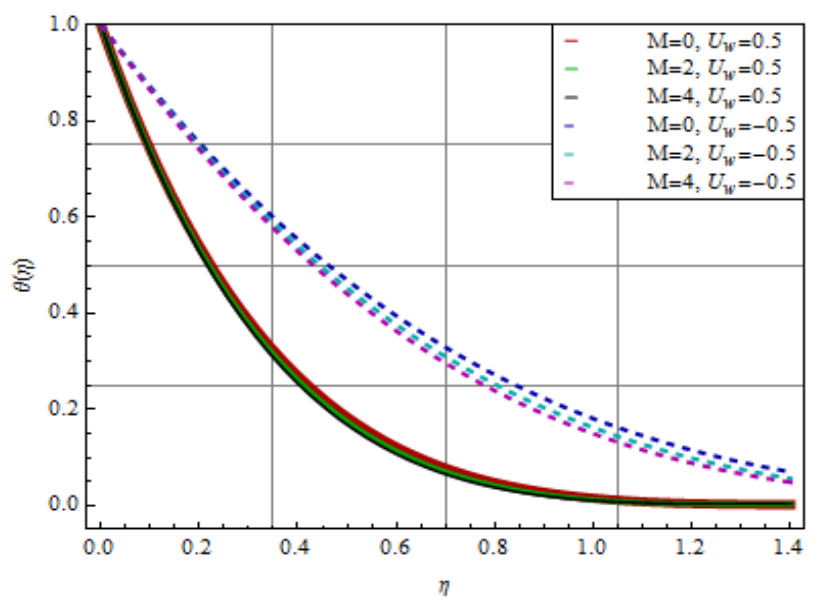

Figure 8. The temperature distribution for alumina-sodium alginate nanofluid for different values of mixed convection and unstediness parameters with $M=1, U_{w}=0.5, \varepsilon=0, P r=$ 6.2, $\varphi=0.2$

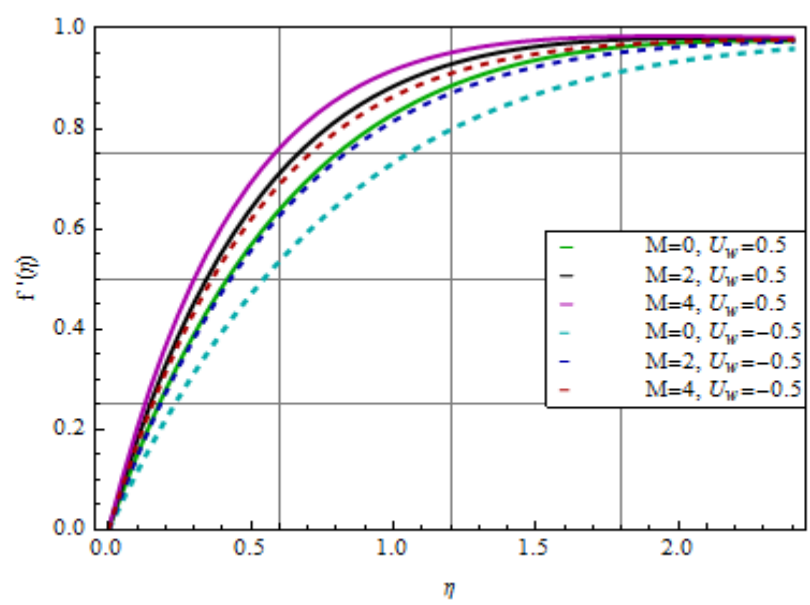

Figure 9. The velocity distribution for alumina-sodium alginate nanofluid for different values of magnetic and velocity ratio parameters with $A=0.5, U_{w}=0.5, \lambda=1, P r=$ 6.2, $\varphi=0.2$

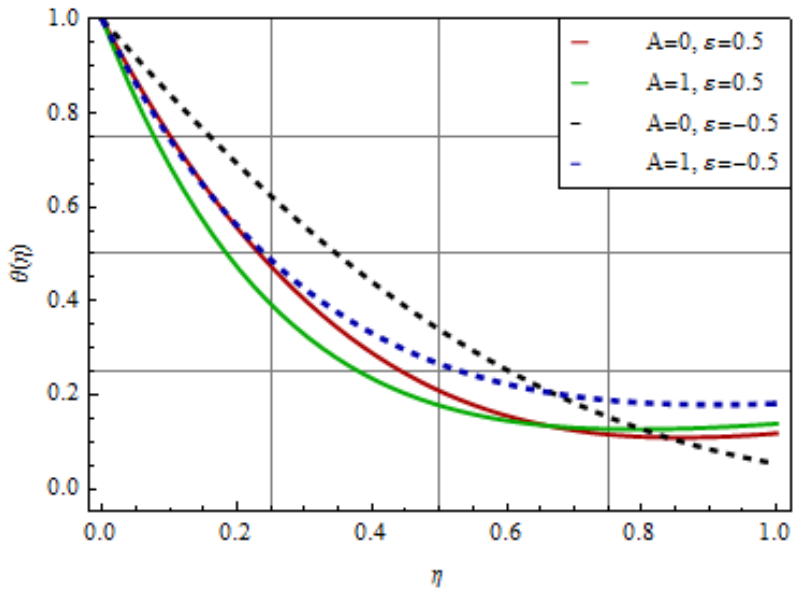

Figure 10. The temperature distribution for alumina-sodium alginate nanofluid for different values of magnetic and velocity ratio parameters with $A=0.5, U_{w}=0.5, \lambda=1, \operatorname{Pr}=$ 6.2, $\varphi=0.2$

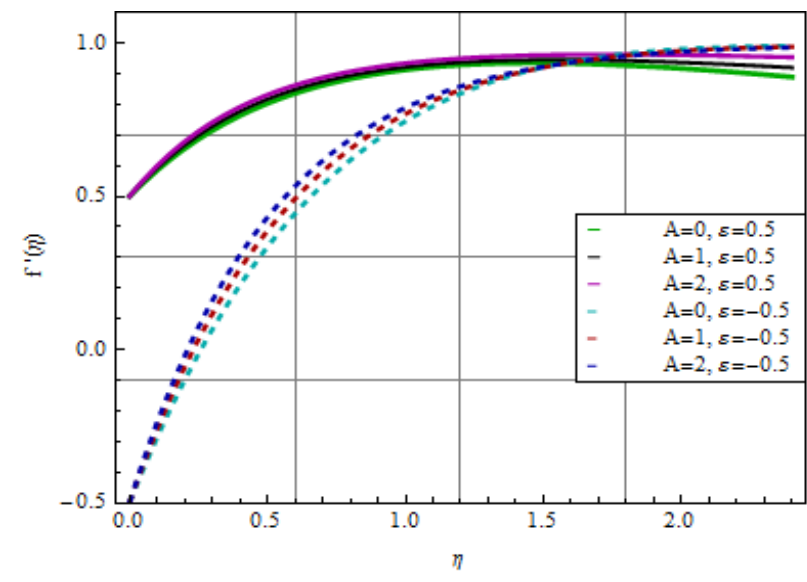

Figure 11. The velocity distribution for alumina-sodium alginate nanofluid for different values of unsteadiness and velocity ratio parameters with $M=1, U_{w}=0.5$, $\lambda=1, \operatorname{Pr}=6.2, \varphi=0.2$

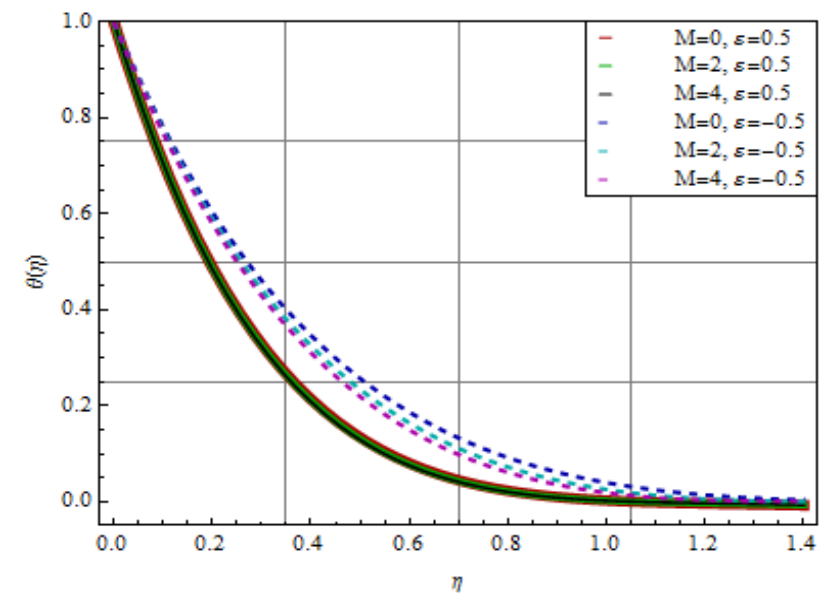

Figure 12. The temperature distribution for alumina-sodium alginate nanofluid for different values of unsteadiness and velocity ratio parameters with $M=1, U_{w}=0.5$, $\lambda=1, \operatorname{Pr}=6.2, \varphi=0.2$ 


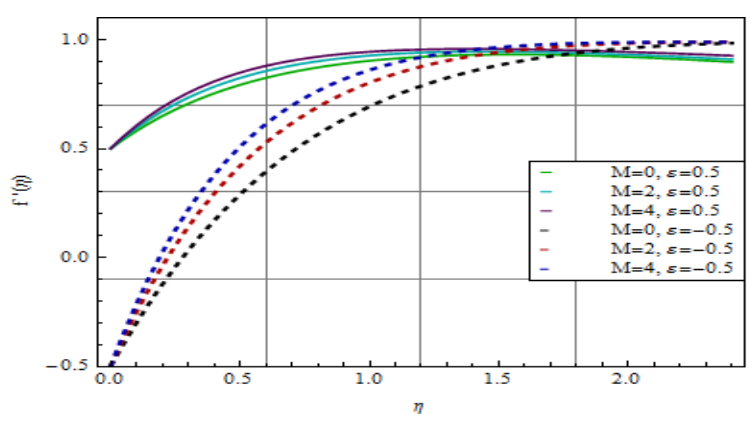

Figure 13. The velocity distribution for alumina-sodium alginate nanofluid for different values of magnetic and wall transpiration parameters with $A=0.5, \varepsilon=0, \lambda=1, P r=6.2$, $\varphi=0.2$

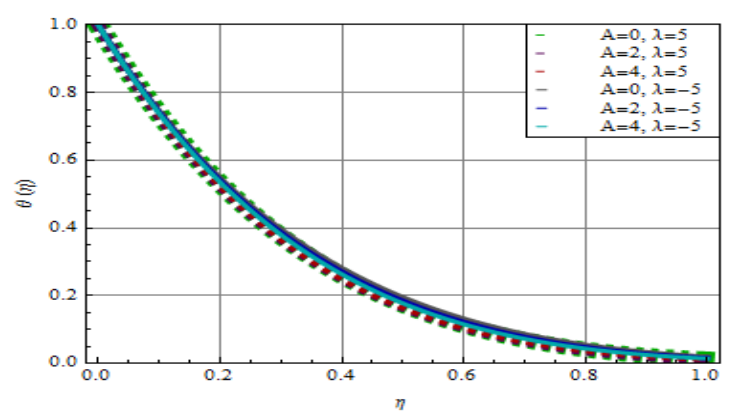

Figure 14. The temperature distribution for alumina-sodium alginate nanofluid for different values of magnetic and wall transpiration parameters with $A=0.5, \varepsilon=0, \lambda=1, P r=6.2$, $\varphi=0.2$

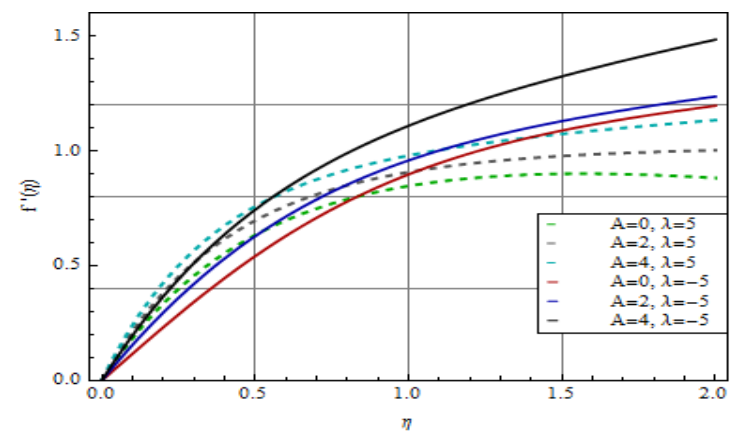

Figure 15. The velocity distribution for alumina-sodium alginate nanofluid for different values of unsteadiness and wall transpiration parameters with $M=1, \varepsilon=0$, $\lambda=1, \operatorname{Pr}=6.2, \varphi=0.2$

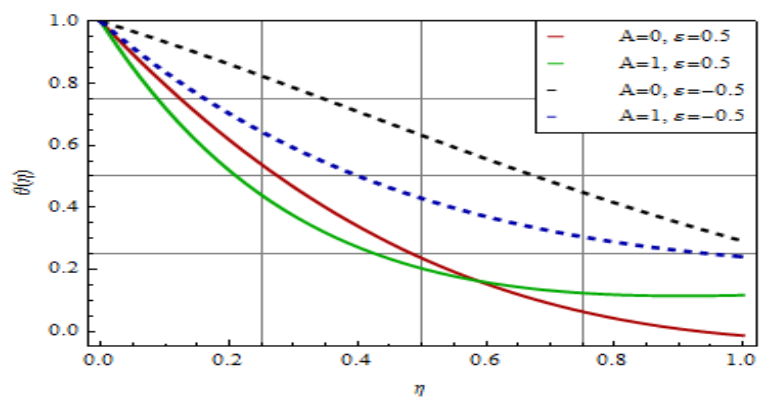

Figure 16. The temperature distribution for alumina-sodium alginate nanofluid for different values of unsteadiness and wall transpiration parameters with $M=1, \varepsilon=0, \lambda=1, P r=$ 6.2, $\varphi=0.2$
Table 5. The effect of the nanoparticle volume fraction on the skin friction coefficient and local Nusselt number for copper-sodium alginate nanofluid with $A=0.5, M=1, U_{w}=$ 0.5

\begin{tabular}{|c|c|c|c|c|c|}
\hline \multirow[b]{2}{*}{ Parameters } & \multicolumn{2}{|c|}{$\lambda=1$} & \multicolumn{3}{|c|}{$\lambda=-1$} \\
\hline & $\varphi$ & $\begin{array}{l}\varepsilon \\
=-0.5\end{array}$ & $\begin{array}{l}\varepsilon \\
=0.5\end{array}$ & $\begin{array}{l}\varepsilon \\
=-0.5\end{array}$ & $\begin{array}{l}\varepsilon \\
=0.5\end{array}$ \\
\hline
\end{tabular}

$\begin{array}{lccccc}R e^{1 / 2} C_{f} & 0.00 & 3.0254 & 1.7859 & 2.8435 & 1.0527 \\ & 0.10 & 4.7987 & 2.0231 & 3.7231 & 1.7868 \\ & 0.20 & 5.6254 & 2.8457 & 5.2428 & 2.0135 \\ N u_{x} R e_{x}^{-1 / 2} & 0.00 & 4.7135 & 5.7134 & 4.4237 & 5.5668 \\ & 0.10 & 4.5387 & 6.0241 & 4.8268 & 6.0258 \\ & 0.20 & 5.0264 & 6.8763 & 5.0214 & 6.7174\end{array}$

Table 6. The effect of the nanoparticle volume fraction on the skin friction coefficient \& local Nusselt number for alumina-sodium alginate nanofluid $A=0.5, M=1, U_{w}=0.5$

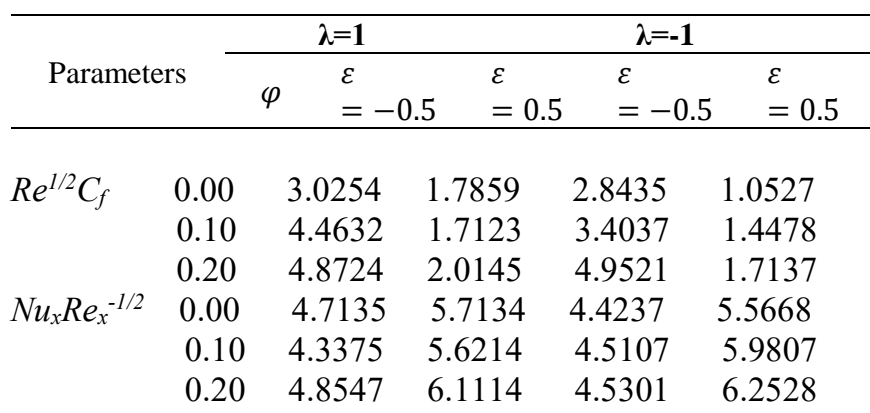

Table 7. The effect of the nanoparticle volume fraction on the skin friction coefficient $\&$ local Nusselt number for titania-sodium alginate nanofluid $A=0.5, M=1, U_{w}=0.5$

\begin{tabular}{|c|c|c|c|c|c|}
\hline \multirow{2}{*}{\multicolumn{2}{|c|}{ Parameters }} & $\lambda=1$ & \multicolumn{3}{|c|}{$\lambda=-1$} \\
\hline & & $\begin{array}{l}\varepsilon \\
=-0.5\end{array}$ & $\begin{array}{l}\varepsilon \\
=0.5\end{array}$ & $\begin{array}{l}\varepsilon \\
=-0.5\end{array}$ & $\begin{array}{l}\varepsilon \\
=0.5\end{array}$ \\
\hline \multirow[t]{3}{*}{$R e^{1 / 2} C_{f}$} & 0.00 & 3.0254 & 1.7859 & 2.8435 & 1.0527 \\
\hline & 0.10 & 4.5874 & 1.9852 & 3.8737 & 1.6897 \\
\hline & 0.20 & 5.2548 & 2.2647 & 5.0021 & 1.8962 \\
\hline \multirow[t]{3}{*}{$N u_{x} R e_{x}^{-1 / 2}$} & 0.00 & 4.7135 & 5.7134 & 4.4237 & 5.5668 \\
\hline & 0.10 & 4.2958 & 5.5237 & 4.2790 & 5.7502 \\
\hline & 0.20 & 4.6250 & 6.0124 & 4.3875 & 6.0125 \\
\hline
\end{tabular}

\section{CONCLUDING REMARKS}

Based on the results and discussions on the MHD nanofluid flow over a stretching/shrinking sheet, the following conclusions have been arrived for alumina-sodium alginate nanofluid:

(1) With increase in magnetic parameter as well as unsteadiness parameter, the velocity increases whereas the temperature decreases.

(2) With increase in Grashof number and mixed convection parameter, the temperature decreases whereas the velocity increases.

(3) With increase in velocity ratio parameter $(\varepsilon)$, the velocity increases whereas the temperature decreases but the magnetic parameter and unsteadiness parameter strongly affect the velocity and temperature distribution in the case of stretching sheet. 
(4) With increase in wall surface transpiration parameter, the velocity increases whereas the temperature decreases but the magnetic parameter and unsteadiness parameter strongly affect the velocity and temperature distribution in the case of suction compared to injection.

(5) The tabulated results presented the highest value of skin friction and local Nusselt number for copper-sodium alginate nanofluid as compared to alumina-sodium alginate and titaniasodium alginate nanofluids.

The results show the simplicity, efficiency and accuracy of HAM for evaluating various kind of rheological problems arising in fluid dynamics

\section{REFERENCES}

[1] Choi SUS. (1995). Enhancing thermal conductivity of fluids with nanoparticles. The Proceedings of ASME International Mechanical Engineering Congress and Exposition, San Francisco, USA Bejan A. Constructal thermodynamics. Constructal Law \& Second Law Conference, Parma S1-S8.

[2] Choi SUS, Zhang ZG, Yu W, Lockwood FE, Grulke EA. (2001). Anomalously thermal conductivity enhancement in nanotube suspensions. Appl Phys Lett 79: 2252-2254. https://doi.org/10.1063/1.1408272

[3] Xuan Y, Li Q. (2000). Heat transfer enhancement of nanofluids. Int $\mathrm{J}$ Heat Fluid Flow 21: 58-64. https://doi.org/10.1016/S0142-727X(99)00067-3

[4] Eastman JA, Choi SUS, Li S, Yu W, Thompson LJ. (2001). Anomalously increased effective thermal conductivities of ethylene glycol-based nanofluids containing copper nanoparticles. Appl Phys Lett 78: 718720. https://doi.org/10.1063/1.1341218

[5] Patel HE, Das SK, Sundarrajan T, Nair AS, George B, Pradeep T. (2003). Thermal conductivities of naked and monolayer protected metal nanoparticle based nanofluids: Manifestation of anomalous enhancement and chemical effects. Appl Phys Lett 83: 2931-2933. https://doi.org/10.1063/1.1602578

[6] Zhou DW. (2004). Heat transfer enhancement of copper nanofluid with acoustic cavitation. Int $\mathrm{J}$ Heat Mass Transfer 47: 3109-3117. https://doi.org/10.1016/j.ijheatmasstransfer.2004.02.018

[7] Hiemenz K. (1911). The boundary layer on a straight circular cylinder immersed in the uniform liquid flow. Dinglers Polytechnisches Journal 326: 321-331. http//dingler.culture.hu-berlin.de/article/pj326/ar326114

[8] Garg VK, Rajagopal KR. (1990). Stagnation-point flow of a non-Newtonian fluid. Mechanics Research Communication 17(6): 415-421. https://doi.org/10.1016/0093-6413(90)90059-L

[9] Wang CY. (2008). Stagnation flow towards a shrinking sheet. International Journal of Non-Linear Mechanics 43(5):

377-382. https://doi.org/10.1016/j.ijnonlinmec.2007.12.021.

[10] Awais M, Hayat T, Mustafa M, Bhattacharyya K, Farooq MA. (2014). Analytic and numeric solutions for stagnation-point flow with melting, thermal-diffusion and diffusion-thermo effects. International Journal of Numerical Methods Heat Fluid Flow 24(2): 438-454. https://doi.org/10.1108/HFF-10-2011-0220

[11] Hayat T, Iqbal Z, Mustafa M, Alsaedi A. (2014). Stagnation-point flow of Jeffrey fluid with melting heat transfer and Soret and Dufour effects. International Journal of Numerical Methods for Heat and Fluid Flow 24(2): 402-418. https://doi.org/10.1108/HFF-02-20120023

[12] Ramachandran N, Chen TS, Armaly BF. (1988). Mixed convection in stagnation flows adjacent to a vertical surface. ASME Journal of Heat Transfer 110: 373-377. https://doi.org/10.1115/1.3250494

[13] Devi CDS, Takhar HS, Nath G. (1991). Unsteady mixed convection flow in stagnation region adjacent to a vertical surface. Heat and Mass Transfer 26(2): 71-79. https://doi.org/10.1007/BF01590239

[14] Ishak A, Nazar R, Pop I. (2007). Mixed convection on the stagnation point flow toward a vertical, continuously stretching sheet. ASME Journal of Heat Transfer 129(8): 1087-1090. https://doi.org/10.1115/1.2737482

[15] Makinde OD, Khan WA, Khan ZH. (2013). Buoyancy effects on MHD stagnation point flow and heat transfer of a nanofluid past a convectively heated stretching/shrinking sheet. International Journal of Heat and Mass Transfer 62: 526-533. https://doi.org/10.1016/j.ijheatmasstransfer.2013.03.049

[16] Tamim H, Dinarvand S, Hosseini R, Pop I. (2014). MHD mixed convection stagnation point flow of a nanofluid over a vertical permeable surface: a comprehensive report of dual solutions. Heat and Mass Transfer 50(5): 639-650. https://doi.org/10.1007/s00231-013-1264-2

[17] Mohammadkazem Sadoughi M, Hosseini F, Shakeri Mohammad reza Azimi (2013). Analytical simulation of MHD nanofluid flow over the horizontal plate. Frontiers in Aerospace Engineering 2(4): 242-246. https://doi.org/10.14355/fae.2013.0204.04

[18] Parsa A, Habib-Olah S. (2016). Analytical solution of MHD flow and heat transfer over a permeable nonlinearly stretching sheet in a porous medium filled by a nanofluid, computational methods for differential equations. 4(1): 70-98.

[19] Nandeppanavar, Mahantesh M. (2016). Flow analysis of MHD Nanofluid due to a stretching sheet with partial slip: An analytical solution. Journal of Nanofluids 5(4): 560567. https://doi.org/10.1166/jon.2016.1239

[20] Rashidi MM, Domairry G, Dinarvand S. (2009). Approximate solutions for the Burger and regularized long wave equations by means of the homotopy analysis method. Commun Nonlinear Sci Numer Simul 14: 708717. https:///10.1016/j.cnsns.2007.09.015

[21] Ziabakhsh Z, Domairry G. (2009). Solution of the laminar viscous flow in a semi-porous channel in the presence of a uniform magnetic field by using the homotopy analysis method. Commun Nonlinear Sci Numer Simul 14: 1284-1294. https://doi.org/10.1016/j.cnsns.2007.12.011

[22] Lok YY, Pop I, Ingham DB, Amin N. (2009). Mixed convection flow of a micropolar fluid near a nonorthogonal stagnation-point on a stretching vertical sheet. International Journal of Numerical Methods for Heat and Fluid Flow 19(3-4): 459-483. https://doi.org/10.1108/09615530910938380

[23] Tiwari RJ, Das MK. (2007). Heat transfer augmentation in a two-sided lid-driven differentially heated square cavity utilizing nanofluids. International Journal of Heat and Mass Transfer 50(9-10): 2002-2018. https://doi.org/10.1016/j.ijheatmasstransfer.2006.09.034 
[24] Oztop HF, Abu-Nada E. (2008). Numerical study of natural convection in partially heated rectangular enclosures filled with nanofluids. International Journal of Heat and Fluid Flow 29(5): 1326-1336. https://10.1016/j.ijheatfluidflow.2008.04.009

\section{NOMENCLATURE}

$A$

$a, b, c$

$a_{g}$

$C_{f}$

$F$

$f(\eta)$

$G r_{y}$

$k$

$M$

$\mathrm{Nu} u_{y}$

$\operatorname{Pr}$

$Q_{w}$

$R e_{y}$

$T$

$T_{0}$

$T_{\infty}$

$T_{w}(y, t)$

$u, v$

$U_{w}$

$U_{w}{ }^{*}$

$V_{\infty}(y, t)$

$V_{w}(y, t)$ unsteadiness parameter

constant

acceleration due to gravity

skin-friction coefficient

magnetic field

dimensionless stream function

local Grashof number

thermal conductivity

magnetic parameter

local Nusselt number

Prandtl number

surface heat flux

local Reynold number

nanofluid temperature

characteristic temperature

ambient temperature

surface temperature

velocity component

wall surface transpiration temperatue

uniform surface mass flux

free stream velocity

surface velocity

\section{Greek symbols}

$\alpha$

$\beta$

$\eta$

$\lambda$

$\mu$

$\phi$

$\Psi$

$\rho$

$\sigma$

$\tau_{w}$

$\theta(\eta)$

$v$

$\varepsilon$

Subscripts

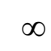

$f$

$N f$

$s$

w

Superscripts thermal diffusivity

thermal expansion coefficient

similarity variable

mixed convection parameter

dynamic viscosity

nanoparticle volume fraction

stream function

fluid density

electrical conductivity

wall shear stress

dimensionless temperature

kinematic viscosity

velocity ratio parameter

ambient condition

base fluid

nanofluid

solid nanoparticle

condition at the surface of wedge

Prime denotes the derivative with respect to $\eta$ 